This study clearly shows that the timing of esophagectomy did not influence overall survival, even though it was associated with increased 90-day mortality. The study suggests that waiting until documented recurrence occurs is not a bad option for patients with a clinical complete response on imaging and endoscopy who are reluctant to undergo surgery. Furthermore, surgeons should be less pressed to operate on patients who appear frail from a nutritional or a medical standpoint after chemoradiation. Waiting will not change these patients' overall likelihood of survival, and optimizing their clinical status may improve outcomes.

Another intriguing finding of the study is the observation that a higher rate of complete pathological response, as assessed after esophagectomy, was seen when esophagectomy was delayed. This raises some important questions: are we operating too early on some of these patients, and would waiting a few extra weeks improve their response to neoadjuvant therapy? Regardless, until better trials are available to address the timing of esophageal resection, we believe that the higher 90-day mortality observed in patients with delayed esophagectomy in this study supports operating within 90 days of completion of chemoradiation therapy whenever possible.

\section{References}

1. van Hagen P, Hulshof MC, van Lanschot JJ, Steyerberg EW, van Berge Henegouwen MI, Wijnhoven BP, et al. Preoperative chemoradiotherapy for esophageal or junctional cancer. N Engl J Med. 2012;366:2074-84.

2. Ajani JA, D'Amico TA, Bentrem DJ, Chao J, Corvera C, Das P, et al. Esophageal and esophagogastric junction cancers, version 2.2019, NCCN Clinical Practice Guidelines in Oncology. J Natl Compr Canc Netw. 2019; 17:855-83.

3. Tepper J, Krasna MJ, Niedzwiecki D, Hollis D, Reed CE, Goldberg R, et al. Phase III trial of trimodality therapy with cisplatin, fluorouracil, radiotherapy, and surgery compared with surgery alone for esophageal cancer: CALGB 9781. J Clin Oncol. 2008;26:1086-92.

4. Mitchell KG, Nelson DB, Corsini EM, Vaporciyan AA, Antonoff MB, Mehran RJ, et al. Morbidity following salvage esophagectomy for squamous cell carcinoma: the MD Anderson experience. Dis Esophagus. July 17, 2019 [Epub ahead of print].

5. Kim JY, Correa AM, Vaporciyan AA, Roth JA, Mehran RJ, Walsh GL, et al. Does the timing of esophagectomy after chemoradiation affect outcome? Ann Thorac Surg. 2012;93:207-12; discussion 212-3.

6. Tessier W, Gronnier C, Messager M, Hec F, Mirabel X, Robb WB, et al. Does timing of surgical procedure after neoadjuvant chemoradiation affect outcomes in esophageal cancer? Ann Thorac Surg. 2014;97:1181-9.

7. Levinsky NC, Wima K, Morris MC, Ahmad SA, Shah SA, Starnes SL, et al. Outcome of delayed versus timely esophagectomy after chemoradiation for esophageal adenocarcinoma. J Thorac Cardiovasc Surg. 2020;159:2555-66.

\title{
Commentary: Delayed resection for esophageal adenocarcinoma
}

\author{
Wayne Hofstetter, MD
}

There have now been multiple retrospective studies examining the potential effect of a delayed operation for esophageal adenocarcinoma after chemoradiation therapy. Uniformly, these studies have shown overall survival results comparable to those of immediate, planned resection, but with some differences in short-term outcomes. ${ }^{1,2}$ Results from these single- and multi-institutional studies are now recapitulated in the study by Levinsky and colleagues ${ }^{3}$

From the Department of Thoracic and Cardiovascular Surgery, The University of Texas MD Anderson Cancer Center, Houston, Tex.

Disclosures: The author has received research support from NeuWave Medical and has served as a consultant for Ethicon.

Received for publication Nov 13, 2019; accepted for publication Nov 14, 2019; available ahead of print Jan 8, 2020.

Address for reprints: Wayne Hofstetter, MD, 1400 Pressler Ave, Unit 1489, Houston, TX 77030 (E-mail: Whofstetter@mdanderson.org).

J Thorac Cardiovasc Surg 2020;159:2568-9

0022-5223/\$36.00

Copyright (c) 2019 by The American Association for Thoracic Surgery

https://doi.org/10.1016/j.jtcvs.2019.11.071

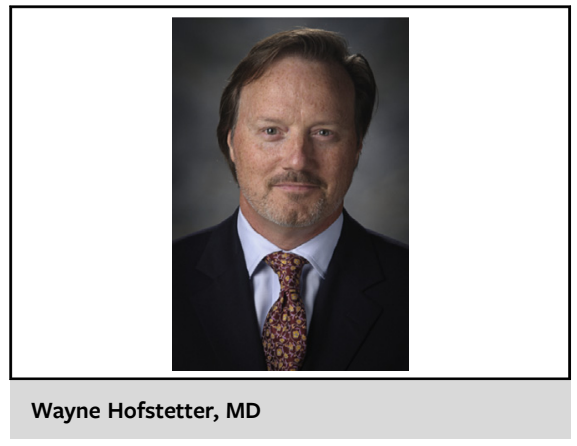

CENTRAL MESSAGE

Patients with persistent or

recurrent esophageal adenocar-

cinoma may undergo delayed

esophagectomy beyond 90 days

after neoadjuvant or definitive

chemoradiation with similar

prognosis compared with timely,

planned esophagectomy. 
derived from the National Cancer Database (NCDB) reported in this issue of the Journal.

What conclusions can be drawn from these data? There are clearly selection biases that affect the decision to operate in a timely versus delayed fashion. None are more apparent than the need/desire to avoid an upfront operation on a sicker patient or one who initially seemed able to tolerate trimodality therapy only to present to the surgeon's office after therapy looking far from an optimal surgical candidate. In those cases, a period of observation and rehabilitation will often lead to an improvement in performance status and/or recognition of persistent disease that would "force" a delayed operation outside the initial 90-day post-chemoradiation (CXRT) period. On the other hand, patients with good performance status who clearly have residual disease after CXRT typically would be offered resection within 90 days. Adding to the heterogeneity, this population differs from those patients who initially had a complete response to therapy and avoided surgery out of choice, risk, or recommendation. These patients would have been under observation but were sent back to a surgeon when the tumor recurred to weigh the risks/benefits of esophagectomy. These are the "true" salvage patients.

Levinsky and colleagues provide evidence that the more remote the surgical date from completion of CXRT, the higher the rate of pathological complete response (pCR). There are several ways to interpret these data, but the most important implication is that these were not all salvage patients. If they were, we would expect a very low rate of $\mathrm{pCR}$ in the final pathology, reflecting the need to undergo esophagectomy only in the situation where tumor was confirmed or highly suspected based on a nondilatable stricture. Critical to making a case for selection bias, these data imply that a reasonable proportion of the delay in surgery within the NCDB data was a result of waiting for patients to recover from CXRT. It would then be a reasonable expectation that patients who had a more delayed course through CXRT and surgery were potentially more susceptible to perioperative events. Kim and colleagues ${ }^{4}$ reported similar findings in a retrospective single-institution analysis of nonsalvage patients who were delayed going to surgery. It is unfortunate that the NCDB data do not offer data on patients' functional decline after CXRT or other surrogates that would allow an imputation of the subgroups. Levinsky and colleagues were left with only the time to surgery as differentiation.

Though there are perioperative event differences, not all of the short-term outcomes described by Levinsky and colleagues are clinically very relevant. Length of stay (LOS) was significantly increased, but the median difference of 1 hospital day with a heavy overlap of the interquartile range does not translate to a clinically relevant endpoint. Nonetheless, as described by the authors, it may be a surrogate endpoint for relatively minor perioperative events that are not otherwise reflected in the data. More importantly, this study reports a significant increase in perioperative mortality, whereas other studies did not. ${ }^{1,2}$ Admitting conjecture, I believe that this is due to heterogeneity in the patient population and not a result of increasing mortality due simply to a time delay from CXRT. The inference of disparity and social differences reported by Levinsky and colleagues seem to be kinetic and attributable variables. Multiple other disease sites (eg, head/neck, rectal) have reported comparable outcomes for early versus late operation after neoadjuvant or definitive CXRT. Yet the concept of radiation "setting in" and increasing the risk of surgery is of concern nonetheless. In fact, the studies of Marks and colleagues ${ }^{1}$ and Markar and colleagues ${ }^{2}$ both call into question the issue of higher mortality surrounding higher radiation doses. ${ }^{1,2}$ These data are not available in the NCDB and are only hypothesis-generating in the other reports. I am interested to see the results of the SANO study, ${ }^{5}$ in which the radiation dose is lower (41.4 Gy), based on the CROSS study. ${ }^{6}$

Finally, the data in the current study are limited to a select number of patients who actually made it to surgery. There is no denominator of total treated, including unresected patients. Because of this, these studies cannot serve as endorsements for selective surgery. Overall, the only conclusion that we should appropriately draw from this study and others like it is that when faced with a situation in which delayed surgery is needed in a patient with esophageal adenocarcinoma, this operation can be done with some confidence and a reasonable prognosis. As to the questions around selective surgery, prospective randomized trials are needed to explore the potential for observation in patients with esophageal adenocarcinomas who are clinical responders.

\section{References}

1. Marks JL, Hofstetter W, Correa AM, Mehran RJ, Rice D, Roth J, et al. Salvage esophagectomy after failed definitive chemoradiation for esophageal adenocarcinoma. Ann Thorac Surg. 2012;94:1126-32; discussion 1132-3.

2. Markar S, Gronnier C, Duhamel A, Pasquer A, Théreaux J, du Rieu MC, et al Salvage surgery after chemoradiotherapy in the management of esophageal cancer: is it a viable therapeutic option? J Clin Oncol. 2015;33:3866-73.

3. Levinsky N, Wima K, Morris MC, Ahmad SA, Shah S, Starnes S, et al. Outcome of delayed versus timely esophagectomy after chemoradiation for esophageal adenocarcinoma. J Thorac Cardiovasc Surg. 2020;159:2555-66.

4. Kim JY, Correa AM, Vaporciyan AA, Roth JA, Mehran RJ, Walsh GL, et al. Does the timing of esophagectomy after chemoradiation affect outcome? Ann Thorac Surg. 2012;93:207-12; discussion 212-3.

5. Noordman BJ, Spaander MCW, Valkema R, Wijnhoven BPL, van Berge Henegouwen MI, Shapiro J, et al. Detection of residual disease after neoadjuvant chemoradiotherapy for oesophageal cancer (preSANO): a prospective multicentre, diagnostic cohort study. Lancet Oncol. 2018;19:965-74.

6. Shapiro J, van Lanschot JJB, Hulshof MCCM, van Hagen P, van Berge Henegouwen MI, Wijnhoven BPL, et al. Neoadjuvant chemoradiotherapy plus surgery versus surgery alone for oesophageal or junctional cancer (CROSS): long-term results of a randomised controlled trial. Lancet Oncol. 2015;16:1090-8. 